\title{
Resistencia antimicrobiana de cepas aisladas de Escherichia coli en alimentos tipo BARF para perros en Lima, 2019
}

\author{
Antimicrobial resistance of isolated strains of Escherichia coli in BARF-type food \\ for dogs in Lima, 2019
}

Kiara Ortega Vassallo ${ }^{1}$, Siever Morales-Cauti ${ }^{1,2}$

\section{Resumen}

\begin{abstract}
El objetivo del presente estudio fue determinar la presencia de cepas de Escherichia coli $(E$. coli) y resistencia antimicrobiana en alimentos tipo BARF (Alimentos crudos biológicamente apropiados) para perros, comercializados en distritos de Lima, Perú. Se analizaron 124 muestras de dietas crudas congeladas pertenecientes a 15 marcas comerciales, cada muestra de distinto lote. Se realizó el aislamiento microbiológico estándar haciendo uso de medios de cultivo MacConkey y EMB, y su identificación por medio de pruebas bioquímicas. Para la serotipificación de E. coli O157 se utilizó Látex test (Oxoid TSMX4147C) con partículas de látex sensibilizadas con anticuerpos reactivos al antígeno somático O157, y para la identificación del antígeno H7, similar Látex test específico (RIM E. coli O157:H7 Latex Test-Oxoid TSMX9410). El 65.3\% (81/124; $\left.\mathrm{IC}_{95 \%}: 56.9-73.7 \%\right)$ de las muestras fueron positivas a $E$. coli. Del total de colonias positivas a $E$. coli, $38.2 \%$ fueron resistentes a los 12 antimicrobianos en estudio.
\end{abstract}

Palabras clave: Escherichia coli, carne, crudo, dieta, contaminación, perro, resistencia

\footnotetext{
${ }^{1}$ Carrera de Medicina Veterinaria y Zootecnia, Universidad Científica del Sur, Lima, Perú

${ }^{2}$ E-mail: sieverm@hotmail.com; https://orcid.org/0000-0002-5396-8889
}

Recibido: 15 de octubre de 2020

Aceptado para publicación: 10 de abril de 2021

Publicado: 23 de junio de 2021

CLos autores. Este artículo es publicado por la Rev Inv Vet Perú de la Facultad de Medicina Veterinaria, Universidad Nacional Mayor de San Marcos. Este es un artículo de acceso abierto, distribuido bajo los términos de la licencia Creative Commons Atribución 4.0 Internacional (CC BY 4.0) [https:// creativecommons.org/licenses/by/4.0/deed.es] que permite el uso, distribución y reproducción en cualquier medio, siempre que la obra original sea debidamente citada de su fuente original 
The aim of this study was to determine the presence of strains of Escherichia coli (E. coli) and antimicrobial resistance in BARF-type foods (Biologically Appropriate Raw Foods) for dogs, marketed in districts of Lima, Peru. In total, 124 samples of frozen raw diets belonging to 15 commercial brands were analysed, each sample from a different batch. The standard microbiological isolation was carried out using MacConkey and EMB culture media, and their identification through biochemical tests. For the serotyping of $E$. coli O157, a Latex test (Oxoid TSMX4147C) was used with latex particles sensitized with antibodies reactive to the somatic antigen O157, and for the identification of the $\mathrm{H} 7$ antigen, a similar specific Latex test (RIM E. coli O157: H7 Latex Test - Oxoid TSMX9410). The 65.3\% (81/124; 95\% CI: 56.9-73.7\%) of the samples were positive for E. coli. Of the total colonies positive for $E$. coli, $38.2 \%$ were resistant to the 12 antimicrobials under study.

Key words: Escherichia coli, meat, raw, diet, contamination, dog, resistance

\section{INTRODUCCIÓN}

La alimentación a base de carne cruda para gatos y perros se incrementado a nivel mundial (Schlesinger y Joffe, 2011). Estas dietas crudas se les denomina «Alimento Crudo Biológicamente Apropiado» (ACBA) o en inglés «Biologically Appropriate Raw Food» (BARF), pudiendo ser elaborados de forma casera y a nivel comercial. En general, se elabora a base de ingredientes crudos derivados de animales, pudiendo incluir músculos, órganos internos, grasa y huesos de mamíferos, peces o aves de corral, así como leche sin pasteurizar, huevos crudos y diversos vegetales (Freeman et al., 2013), y son vendidos en forma fresca o congelada (Freeman et al., 2013; Dadd et al., 2019).

Entre los beneficios de la alimentación con este tipo de dietas se encuentra la calidad nutricional, mejor palatabilidad, dientes y encías más sanas, pelaje más brillante, mejor microbiota intestinal, heces más duras y mejora de la energía del animal (Craig, 2020). Este tipo de alimentación cruda promueve un crecimiento más equilibrado de comunidades bacterianas y, por ende, un cambio positivo para las funciones intestinales saludables (Sandri et al., 2017); sin embargo, los perros poseen un mayor riesgo de infecciones oportunistas (Kim et al., 2017).

Morelli et al. (2019) encontraron que el $60 \%$ de los propietarios eligieron este tipo de alimentación por información encontrada en el Internet y que el $94 \%$ considera el BARF como una alimentación segura. No obstante, Anturaniemi et al. (2019) reportó la trasmisión de patógenos desde el alimento crudo para mascotas a los humanos, donde Escherichia coli (E. coli) fue el agente causal. En la actualidad, existen diversos estudios que indican los riesgos potenciales para la mascota asociados al uso de la alimentación con BARF (Aquino, 2020), siendo una minoría de estas dietas elaboradas por médicos veterinarios especializados en el área de nutrición (Morelli et al., 2019). Se ha reportado que hasta el $95 \%$ de recetas elaboradas en casa presentan por lo menos un nutriente por debajo del mínimo dado por la Association of American Feed Control Officials (AAFCO) y que hasta $83 \%$ contenía múltiples deficiencias (Stockman et al., 2013). 
El tema más discutido en la actualidad es el riesgo para la salud animal y la salud pública, debido a la posible contaminación de BARFs con agentes patógenos. Van Bree et al. (2018) evaluaron 35 dietas comerciales congeladas de ocho empresas a base de carne cruda, aislando E. coli $\mathrm{O} 157: \mathrm{H} 7$ en el 23\%, E. coli productoras de betalactamasas de espectro extendido en el $80 \%$, además de contaminaciones con Listeria monocytogenes, Salmonella spp y parásitos como Sarcocystis cruzi y Toxoplasma gondi; evidenciando la presencia de múltiples agentes patógenos en las dietas BARFs.

E. coli es un patógeno que toma gran relevancia en enfermedades trasmitidas por alimentos (ETA). Es un bacilo gramnegativo, anaerobio facultativo, de la familia Enterobacteriaceae, la cual puede encontrarse como miembro comensal de la microbiota intestinal de animales y humanos; sin embargo, algunas cepas resultan ser patógenos causales de enfermedades intestinales y extra intestinales (Mainil, 2013). La presencia de E. coli patógena puede generar en el perro una colibacilosis, produciendo daño a nivel del tracto gastrointestinal (Zotta et al., 2015).

Los serotipos de E. coli patógenos que causan daño a nivel intestinal ocasionando diarreas en el hospedador se les denomina $E$. coli diarreogénica, los cuales a su vez se subdividen en seis patotipos: enterotoxigénica (ECET), enteropatógena (ECEP), enteroinvasiva (ECEI), enteroagregativa (ECEA), enterohemorrágica (ECEH) y de adherencia difusa (ECAD) (Rodríguez-Angeles, 2002; Mainil, 2013). La ECEH también es conocida como productora de toxina Vero o toxina semejante a Shiga (VTEC o STEC); siendo la cepa más frecuente de este subgrupo la $E$. coli serotipo O157:H7 (Saeedi et al., 2017), Esta cepa tiene gran relevancia en investigaciones a nivel mundial, debido a su alta patogenicidad, y su relación en cuanto a su presencia en productos crudos de consumo humano y animal, teniendo al bovino como su principal reservorio (Rodríguez-Angeles, 2002; Saeedi et al., 2017).

La resistencia bacteriana a antimicrobianos adquiere importancia debido a que puede ser adquirida por las mascotas a través del consumo de productos BARFs contaminados. Una investigación en Lima, Perú, reveló un elevado nivel de resistencia de antimicrobianos en carne de pollo, res y cerdo de mercados de abasto (Ruiz-Roldan et al., 2018). Asimismo, se ha reportado un alto porcentaje de resistencia a antimicrobianos de bacterias presentes en dietas crudas, especialmente a cefalosporinas de tercera generación y productoras de betalac-tamasas de expectro extencido (BLEEs) (NueschIderbinen et al., 2019).

Se plantea que los médicos veterinarios deben tomar un rol más activo con relación a la toma de decisión de los dueños en cuanto a la alimentación de su mascota y los riesgos que implican las dietas tipo BARF. Para que la intervención del veterinario tenga más relevancia, es importante que posean los conocimientos y la data previa necesaria para entender en su totalidad la situación epidemiológica y poder asesorar correctamente a los propietarios (Hinney, 2018). De esta manera, el objetivo del presente estudio fue determinar la presencia de E. coli $\mathrm{O} 157: \mathrm{H} 7$ y resistencia antimicrobiana en alimentos tipo BARF para perros, que se comercializan en los distritos de Lima, Perú.

\section{Materiales y MéTodos}

\section{Población y Muestra}

La población del estudio comprendió unidades de dietas comerciales BARF congeladas, que son comercializadas en Lima Metropolitana y que fueron obtenidas entre julio y diciembre de 2019. El tamaño de muestra se estimó utilizando $23 \%$ de prevalencia obtenida de un estudio similar realizado en Europa (Van Bree et al., 2018), debido a que 
no existen estudios similares realizados en el Perú o Latinoamérica. Se empleó un $95 \%$ de confiabilidad y $5 \%$ de error; utilizándose la siguiente fórmula de presencia o ausencia (Thrusfield, 2005): $\mathrm{n}=\left(1-(1-\mathrm{NC})^{1 / \mathrm{d}}\right) *(\mathrm{n}-(\mathrm{d}-$ 1)/2), donde $\mathrm{NC}=$ Nivel de confianza, $\mathrm{N}=$ Tamaño de la población de referencia, $\mathrm{d}=$ Prevalencia esperada, $\mathrm{n}=$ Tamaño mínimo de muestra. El tamaño muestral mínimo obtenido fue de 120 muestras; sin embargo, se evaluaron 124 muestras, las cuales fueron obtenidas de 15 marcas comerciales disponibles en la ciudad.

\section{Muestreo y Lugar de Estudio}

El estudio fue de tipo observacional, prospectivo, descriptivo y transversal. Se realizó un muestreo aleatorio en el universo de muestras disponibles en comercialización. Se seleccionó como muestras a aquellos alimentos tipo BARF para perros, fabricados a base de carne cruda de origen animal y de vegetales crudos, los cuales se encontraron de forma congelada. Estos productos eran comercializados vía delivery y/o en tiendas veterinarias.

El procesamiento de las muestras se realizó en el Laboratorio de Microbiología de la Universidad Científica del Sur, localizada en Villa el Salvador, Lima, Perú. Las muestras fueron almacenadas en congelamiento $\left(-18^{\circ} \mathrm{C}\right)$ y llevadas al laboratorio dentro de cajas térmicas, conservando la cadena de frío. Una vez recibidas en el laboratorio fueron descongeladas y llevadas a temperatura ambiente.

\section{Identificación de Escherichia coli}

Los alimentos fueron homogenizados. La identificación de $E$. coli se realizó de forma convencional (INS, 2001). Para esto, se tomaron $10 \mathrm{~g}$ por muestra homogenizada $\mathrm{y}$ puestas en un enriquecimiento previo en caldo Tripticasa de soya a $37^{\circ} \mathrm{C}$ por $24 \mathrm{~h}$, luego fueron sembradas en agar MacConkey y EMB (Eosina y Azul de Metileno agar) e incubadas a $37^{\circ} \mathrm{C}$ por $24 \mathrm{~h}$. Las cepas sospechosas de $E$. coli fueron confirmadas a través de las pruebas bioquímicas: LIA (Lisina Hierro agar), citrato de Simmons, SIM (sulfidrilo-indol-motilidad), urea y TSI (Triple Sugar Iron agar), cultivadas a $37^{\circ} \mathrm{C}$ por 24 h. Además, algunas de las cepas confirmadas se les hizo la tinción Gram para ser observadas al microscopio.

\section{E. coli serotipo 0157:H7}

Las cepas compatibles con E. coli fueron subcultivadas en agar MacConkey sorbitol. Las colonias que no fermentaron sorbitol (colonias incoloras) fueron aisladas y sometidas al E. coli O157 Latex Test (Oxoid TSMX4147C), el cual posee partículas de látex sensibilizadas con anticuerpos específicos reactivos al antígeno somático O157. Para la serotipificación se utilizó RIM E. coli O157:H7 Latex Test (Oxoid TSMX9410), con similar mecanismo a la prueba antes mencionada, resultando positivo la aglutinación.

\section{Resistencia a Antimicrobianos}

Las cepas positivas a E. coli confirmadas mediante las pruebas bioquímicas fueron almacenadas en crioviales con caldo Tripticasa de soya hasta ser nuevamente analizadas. Se determinó la sensibilidad antimicrobiana frente a 12 fármacos, mediante el método de difusión en disco (método KirbyBauer), siguiendo el modelo de la guía CLSI (Clinical and Laboratory Standards Institute) (CLSI, 2018). Los antimicrobianos fueron: Ac. Nalidíxico $(30 \mu \mathrm{g} / \mathrm{ml})$, Azitromicina $(15 \mu \mathrm{g} /$ $\mathrm{ml})$, Imipenem (10 $\mu \mathrm{g} / \mathrm{ml})$, Cotrimoxazol (25 $\mu \mathrm{g} / \mathrm{ml})$, Gentamicina $(10 \mu \mathrm{g} / \mathrm{ml})$, Cloranfenicol $(30 \mu \mathrm{g} / \mathrm{ml})$, Amoxicilina con Ácido clavulánico (30 $\mu \mathrm{g} / \mathrm{ml})$, Ciprofloxacina $(5 \mu \mathrm{g} / \mathrm{ml})$, Furazolidona $(100 \mu \mathrm{g} / \mathrm{ml})$, Nitrofurantoina $(300 \mu \mathrm{g} / \mathrm{ml})$, Ampicilina $(10 \mu \mathrm{g} / \mathrm{ml}) \mathrm{y}$ Tetraciclina $(30 \mu \mathrm{g} / \mathrm{ml})$. Las cepas fueron sembradas en agar Muller Hinton, posteriormente se ubicaron seis discos en cada placa, y se incubaron nuevamente a $37^{\circ} \mathrm{C}$ por $24 \mathrm{~h}$. 
Cuadro 1. Frecuencia de muestras positivas a $E$. coli según el número de muestras por marca de alimento tipo BARF comerciales en Lima (2019)

\begin{tabular}{|c|c|c|c|c|c|}
\hline \multirow{3}{*}{ Marca } & \multirow{3}{*}{$\begin{array}{c}\text { Muestras } \\
\text { (n) }\end{array}$} & \multirow{2}{*}{\multicolumn{2}{|c|}{ Positivos }} & \multicolumn{2}{|c|}{ IC 95\% } \\
\hline & & & & \multirow{2}{*}{ Mínimo } & \multirow{2}{*}{ Máximo } \\
\hline & & $\mathrm{n}$ & $\%$ & & \\
\hline $\mathrm{D}$ & 10 & 10 & 100.0 & 100.0 & 100.0 \\
\hline $\mathrm{L}$ & 9 & 9 & 100.0 & 100.0 & 100.0 \\
\hline M & 9 & 9 & 100.0 & 100.0 & 100.0 \\
\hline $\mathrm{C}$ & 2 & 2 & 100.0 & 100.0 & 100.0 \\
\hline $\mathrm{F}$ & 2 & 2 & 100.0 & 100.0 & 100.0 \\
\hline $\mathrm{N}$ & 9 & 8 & 88.9 & 68.4 & 100.0 \\
\hline $\mathrm{J}$ & 7 & 6 & 85.7 & 59.8 & 100.0 \\
\hline$\tilde{N}$ & 10 & 8 & 80.0 & 55.2 & 100.0 \\
\hline A & 10 & 7 & 70.0 & 41.6 & 98.4 \\
\hline $\mathrm{G}$ & 9 & 6 & 66.7 & 35.9 & 97.5 \\
\hline I & 9 & 4 & 44.4 & 12.0 & 76.9 \\
\hline $\mathrm{K}$ & 9 & 4 & 44.4 & 12.0 & 76.9 \\
\hline B & 10 & 3 & 30.0 & 1.6 & 58.4 \\
\hline $\mathrm{H}$ & 9 & 2 & 22.2 & 0 & 49.34 \\
\hline $\mathrm{E}$ & 10 & 1 & 10.0 & 0 & 28.6 \\
\hline Total & 124 & 81 & 65.3 & 57.0 & 73.7 \\
\hline
\end{tabular}

\section{Resultados}

El $65.32 \%(81 / 124)$ de las muestras fueron positivas a $E$. coli $\left(\mathrm{IC}_{95 \%}: 56.9-73.7 \%\right)$, sin que se haya identificado alguna cepa como E. coli serotipo O157:H7. Los resultados indican que 8 de las 15 marcas comerciales presentaron no menos del $80 \%$ de muestras positivas a $E$. coli (Cuadro 1).

De los insumos, la carne de res fue el tipo de proteína más utilizada y evaluada, encontrando $73.3 \%$ (22/30) de muestras positivas a $E$. coli. Asimismo, de las muestras con pollo el $38.1 \%$ resultó positivo $(8 / 21)$ y las de pavo $85.7 \%$ (12/14) (Cuadro 2).
Del total de muestras positivas a E. coli, $38.2 \%$ de las colonias evaluadas resultaron resistentes y el 17.0\% fueron intermedias. En ampicilina, cotrimoxazol, ácido nalidixico y cloranfenicol se encontró un porcentaje de resistencia superior al $50 \%$, mientras que para la tetraciclina, furazolidona e imipenem fue superior al $30 \%$. Solo una colonia evaluada contra azitromicina resulto resistente (Cuadro 3).

\section{Discusión}

El presente estudio no identificó cepas E. coli $\mathrm{O} 157: \mathrm{H} 7$ en las muestras analizadas; sin embargo, a nivel de Latinoamérica se han 
Cuadro 2. Frecuencia de muestras positivas a E. coli según el tipo de proteína utilizada en el alimento tipo BARF comercial para alimento de perros (Lima, 2019)

\begin{tabular}{|c|c|c|c|c|c|}
\hline \multirow{3}{*}{$\begin{array}{l}\text { Tipo de proteína } \\
\text { animal }\end{array}$} & \multirow{3}{*}{$\begin{array}{l}\text { Muestras } \\
\text { (n) }\end{array}$} & \multicolumn{2}{|c|}{ Positivos } & \multicolumn{2}{|c|}{ IC 95\% } \\
\hline & & & & \multirow{2}{*}{ Mínimo } & \multirow{2}{*}{ Máximo } \\
\hline & & $\mathrm{n}$ & $\%$ & & \\
\hline Res & 30 & 22 & 73.3 & 57.5 & 89.2 \\
\hline Res y pavo & 22 & 13 & 59.1 & 38.6 & 79.6 \\
\hline Pollo & 21 & 8 & 38.1 & 17.3 & 58.9 \\
\hline Pavo & 14 & 12 & 85.7 & 67.4 & 100.0 \\
\hline Res y pollo & 11 & 8 & 72.7 & 46.4 & 99.0 \\
\hline Res y cerdo & 9 & 7 & 77.8 & 50.6 & 100.0 \\
\hline Pollo, pavo y pato & 5 & 5 & 100.0 & 100.0 & 100.0 \\
\hline Pavo y pato & 4 & 1 & 25.0 & 0 & 67.4 \\
\hline Res y equino & 3 & 3 & 100.0 & 100.0 & 100.0 \\
\hline Cordero & 3 & 1 & 33.3 & 0 & 86.7 \\
\hline Res, pavo y pollo & 1 & 1 & 100.0 & 100.0 & 100.0 \\
\hline Pavo y pollo & 1 & 0 & 0 & 0 & 0 \\
\hline Total & 124 & 81 & 65.3 & 57.0 & 73.7 \\
\hline
\end{tabular}

reportado prevalencias de 1.4 y $3.5 \%$ en Argentina y Brasil, respectivamente de E. coli O157:H7 productora de toxina Shiga en productos cárnicos de res (Morato et al., 2007; Jure et al., 2015), y 1.5\% en carne molida en Perú (Mendez et al., 2013). A nivel internacional, se ha reportado en Turquía y Etiopia 1.9 y $10.2 \%$ de muestras positivas de carne de pollo y en carnes, respectivamente (Bekele et al., 2014; Dincoglu y Gunulalan, 2016), en tanto que en España en preparados cárnicos y en Sudáfrica en carne seca, carne picada y embutidos se le aisló en 19.4 y $2.8 \%$ de las muestras, respectivamente (Abong'o y Momba, 2009; Ripodas et al., 2017). Los reportes de hallazgos en diversos países confirman la alta posibilidad y el potencial riesgo de la presencia de este serotipo; por lo que, el uso de una técnica más sensible, otras cir- cunstancias de muestreo, o un mayor número de muestras analizadas podría haber permitido el aislamiento de cepas E. coli O157:H7 en el presente estudio.

El 65.3\% (81/124) de las muestras positivas a $E$. coli $\left(\mathrm{IC}_{95 \%}: 56.9-73.7 \%\right)$ en el presente estudio se encuentra dentro del rango de resultados reportados en muestras de mercados de abasto de la ciudad de Lima. Así, Mendez et al. (2013) reportó 87.2\% positivos en muestras de carne molida y Lucas et al. (2016) de $42 \%$ de puestos de venta de carne de pollo. Esto representa un riesgo importante, pues la mayoría de los insumos necesarios para la elaboración del BARF son adquiridos en este tipo de mercados. A su vez, Weese et al. (2005) reportaron que los coliformes fueron hallados en todas las mues- 
Cuadro 3. Frecuencia ${ }^{1}$ de la resistencia a antimicrobianos de las cepas de Escherichia coli aislados en BARFs comerciales de Lima (2019)

\begin{tabular}{lcccccc}
\hline \multirow{2}{*}{ Antimicrobiano } & \multicolumn{2}{c}{ Sensible } & \multicolumn{2}{c}{ Intermedio } & \multicolumn{2}{c}{ Resistente } \\
\cline { 2 - 7 } & $\mathrm{n}$ & $\%$ & $\mathrm{n}$ & $\%$ & $\mathrm{n}$ & $\%$ \\
\hline Ampicilina & 7 & 10.4 & 14 & 20.9 & 46 & 68.7 \\
Cloranfenicol & 3 & 4.5 & 19 & 28.4 & 45 & 67.2 \\
Ácido nalidíxico & 10 & 14.9 & 16 & 23.9 & 41 & 61.2 \\
Cotrimoxazol & 23 & 34.3 & 5 & 7.5 & 39 & 58.2 \\
Tetraciclina & 30 & 44.8 & 4 & 6 & 33 & 49.3 \\
Imipenem & 22 & 32.8 & 20 & 29.9 & 25 & 37.3 \\
Furazolidona & 26 & 38.8 & 19 & 28.4 & 22 & 32.8 \\
Ciprofloxacina & 43 & 64.2 & 4 & 6 & 20 & 29.9 \\
Gentamicina & 46 & 68.7 & 8 & 11.9 & 13 & 19.4 \\
Nitrofurantoina & 35 & 52.2 & 21 & 31.3 & 11 & 16.4 \\
Amoxicilina Ác. & 49 & 73.1 & 7 & 10.5 & 11 & 16.4 \\
clavulánico & 66 & 98.5 & 0 & 0 & 1 & 1.5 \\
Azitromicina & 360 & 44.8 & 137 & 17.0 & 307 & 38.2 \\
\hline Total & & &
\end{tabular}

${ }^{1}$ Porcentajes calculados sobre el número total de colonias positivas evaluadas

tras de dieta cruda comercial que evaluaron, donde $E$. coli fue identificada en el $64 \%$, similar al reporte del presente estudio. En este sentido, Van Bree et al. (2018) reportaron un porcentaje aún más elevado $(86 \%)$ de aislamiento de E. coli en dietas crudas comerciales congeladas.

En el total de muestras de cinco marcas se aisló E. coli como contaminante, pese a que las muestras provenían de lotes diferentes. Esto posiblemente se debe a que los insumos utilizados ya se encontraban contaminados desde el puesto de venta donde fueron adquiridos (mercados de abasto), o que en uno o varios puntos de la cadena de producción haya un mal manejo o equipos contaminados, representando un riesgo permanente de contaminación.
El gran porcentaje de positivos (65.3\%) a $E$. coli puede deberse a que las dietas BARF son productos altamente manipulados, incrementando de esta manera el riesgo a la exposición de agentes patógenos. La carne molida, base de la mayoría de las dietas evaluadas, por su gran manipulación es aún más susceptible a la contaminación microbiana (Mendez et al., 2013).

La carne de res fue el tipo de proteína más utilizado en la preparación de las dietas BARF, y pese a que no se encontró $E$. coli O157:H7, el bovino es considerado como su principal reservorio. No obstante, el 73.3\% de muestras positivas a $E$. coli en alimentos con base de carne de res fue encontrado en el estudio, valor superior al $65.7 \%$ reportado por Strohmeyer et al. (2006). Por otro lado, 
la carne de aves de corral, ingredientes muy utilizados en las dietas crudas, se convierte en posible fuente de contaminación, debido a una potencial contaminación cruzada durante su manipulación (Lucas et al., 2016).

No solo los productos de origen animal son una fuente de contaminación. Un estudio realizado en Lima por Muñoz et al. (2013), reportó que el $18.9 \%$ de verdura fresca presentó niveles de $E$. coli fecales superior a lo establecido como apto por la ICMSF (International Commission on Microbiological Specification for Foods), siendo la espinaca la más contaminada.

Se encontró $38.2 \%$ de nivel de resistencia a 12 antimicrobianos, porcentaje mayor al 23\% reportado por Nilsson (2015) en muestras de dietas crudas para perros. Ampicilina fue el antimicrobiano con el mayor nivel de resistencia $(68.67 \%)$, porcentaje inferior al 94.6\% reportado en carne de pollo y del $95.4 \%$ en carne de cerdo, pero superior al $44 \%$ en carne de res (Ruiz-Roldan et al., 2018). En segundo nivel de resistencia está el cloranfenicol (67.26\%), porcentaje inferior al $82.1 \%$ encontrado en carne de pollo, pero superior al $4 \%$ en carne de res y de $54.5 \%$ en carne de cerdo (Ruiz-Roldan et al., 2018). Los resultados indican que este tipo de dietas puede ser una fuente potencial de agentes resistente a fármacos de primera elección (Nilsson, 2015).

A diferencia de los alimentos de consumo humano, no hay una autoridad especifica que sea responsable del monitoreo microbiológico continuo de los alimentos para perros a base de productos crudos como las dietas tipo BARF (Strohmeyer et al., 2006). En el Perú, el Servicio Nacional de Sanidad Agraria (SENASA), es la entidad encargada de la inocuidad agroalimentaria, mas no posee reglamentación específica para las dietas crudas para mascotas; exponiendo a estos animales a este tipo de prácticas.
La ausencia de positivos para la cepa O157:H7 en este estudio pudo ser distinto con la utilización de otro tipo de serotipificación, tal como la separación inmunomagnética (Jure et al., 2015), test de concentración por inmunocaptura (Marzocca et al., 2006) o molecularmente mediante PCR (Mora et al., 2006; Mendez et al., 2013; Lucas et al., 2016), reportes que respaldan la presencia de esta bacteria en diversos productos cárnico.

\section{Conclusiones}

- No se aisló E. coli serotipo O157:H7 en las muestras de alimentos tipo BARF destinadas a la alimentación de perros en la ciudad de Lima, Perú.

- $\quad$ En el 65.3\% (81/124) de las muestras analizadas de alimento tipo BARF se aisló E. coli.

- $\quad$ El 100\% de las cepas presentaron resistencia a al menos a un antimicrobiano, siendo la resistencia a la ampicilina la frecuencia más alta $(68.7 \%, 46 / 67)$ y la frecuencia más baja correspondió a la azitromicina con un $1.5 \%(1 / 67)$.

\section{Literatura Citada}

1. Abong'o BO, Momba MN. 2009. Prevalence and characterization of Escherichia coli $\mathrm{O} 157: \mathrm{H} 7$ isolates from meat and meat products sold in Amothe district, Eastern Cape province of South Africa. Food Microbiol 26: 173-176. doi: doi:10.1016/j.fm.2008.10.001

2. Anturaniemi J, Barrouin-Melo SM, Zaldivr-López, S, Sinkko H, HielmBjorkman A. 2019. Owners' perception of acquiring infections through raw pet food: a comprehensive internet-basedsurvey. Vet Rec 185: 658. doi:10.1136/ vetrec-2018-105122 
3. Aquino W. 2020. Evaluación bromatológica y microbiológica de cuatro marcas comerciales de alimento BARF para caninos. Tesis de Médico Veterinario y Zootecnista. Guayaquil: Univ. de Guayaquil. $125 \mathrm{p}$.

4. Bekele T, Zewde G, Tefera G, Feleke A, Zerom K. 2014. Escherichia coli O157:H7 in raw meat in Addis Ababa, Ethiopia: prevalence at an abattoir and retailers and antimicrobial susceptibility. Int J Food Contam 1:4. doi: 10.1186/ s40550-014-0004-9

5. [CLSI] Clinical and Laboratory Standards Institute. 2018. Performance standards for antimicrobial disk susceptibility tests. $13^{\text {th }}$ ed. CLSI Standard M02. Wayne, PA: CLSI. 92 p.

6. Craig JM. 2020. Raw feeding in dogs and cats. Companion Animal Pract 24: 578-584. doi: 10.12968/coan.2018.0068

7. Dadd S, Barry M, Grant C, Verbrugghe A. 2019. Abnormal bone mineralization in a puppy fed an imbalanced raw meat homemade diet diagnosed and monitored using dualenergy X ray absorptiometry. J Anim Physiol Anim Nutr 00: 1-8. doi: 10.1111/ jpn. 13118

8. Dincoglu AH, Gunulalan Z. 2016. Determination of Escherichia coli O157:H7 in chicken meats sold in Sanliurfa region. Mnnas J Engin 4: 52-68.

9. Freeman LM, Chandler ML, Hamper $B A$, Weeth LP. 2013. Current knowledge about the risks and beneûts of raw meat-based diets for dogs and cats. J Am Vet Med Assoc 243: 1549-1558. doi: 10.2460/javma.243.11.1549

10. Hinney B. 2018. The trend of raw meat-based diets: risks to people and animals. Vet Rec 182: 47-49. doi: 10.1136/ vr.k7

11. [INS] Instituto Nacional de Salud. 2002. Manual de procedimientos bacteriológicos en infecciones intrahospitalarias. 89 p. [Internet]. Disponible en: https://cdn.www.gob.pe/uploads/document/file/342324/Manual_-de_proce-
dimientos_-bacteriol\%C3\%-B3gicos_en_infecciones_intra-hospitalarias20190716-19467-1s8gfxd.-pdf

12. Jure MA, Condorí MS, Perez G, Catalan M, López A, Zolezzi G, et al. 2015. Aislamiento y caracterización de Escherichia coli $\mathrm{O} 157$ en productos cárnicos bovinos y medias reses en la provincia de Tucumán. Rev Argent Microbiol 47: 125-131. doi: 10.1016/ j.ram.2015.03.006

13. Kim J, An J, Kim W, Lee S, Cho S. 2017. Differences in the gut microbiota of dogs (Canis lupus familiaris) fed a natural diet or a commercial feed revealed by the Illumina MiSeq platform. Gut Pathog 9: 68. doi: 10.1186/s13099017-0218-5

14. Lucas JR, Morales S, Salazar EP, Eslava C, Alvarado D. 2016. Contaminación por Escherichia coli shigatoxigénica en puestos de expendio de carne de pollo en un distrito de Lima. Rev Inv Vet Perú 27: 618-625. doi: 10.15381/ rivep.v27i3.12000

15. Mainil J. 2013. Escherichia coli virulence factors. Vet Immunol Immunopathol 152: 2-12. doi: 10.1016/j.vetimm.2012.09.032

16. Mendez CR, Vergaray G, Morante HY, Flores PR, Gamboa RA. 2013. Aislamiento y caracterización de Escherichia coli $\mathrm{O} 157: \mathrm{H7}$ a partir de carne molida de bovino en Lima-Perú. Rev Peru Biol 20: 159-154. doi: 10.15381/rpb.v20i2.-2680

17. Mora A, Leon SL, Blanco M, Blanco JE, Lopez C, Dahbi G, et al. 2007. Phages types, virulence genes and PFGE profiles of Shiga toxin-producing Escherichia coli $\mathrm{O} 157: \mathrm{H} 7$ insolated from raw beef, soft cheese and vegetables in Lima (Peru). J Food Ind Microbiol 114: 204-210. doi: 10.1016/ j.ijfoodmicro.2006.09.009

18. Morelli G, Bastianello S, Castellani P, Ricci R. 2019. Raw meat-based diets for dogs: survey of owners' motivations, attitudes and practices. BMC Vet Res 15:74. doi: 10.1186/s12917-019-1824-x 
19. Morato AM, Simoes M, Irino K, Tardelli TA, Cabilio BE. 2007. Prevalence and characteristics of shiga toxinproducing Escherichia coli (stec) strains in ground beef in São Paulo, Brazil. Braz J Microbiol 38: 553-556. doi: 10.1590/S1517-83822007000300032

20. Marzocca MA, Marucci PL, Sica MG, Álvarez EE. 2006. Detección de Escherichia coli O157:H7 en carne picada fresca y hamburguesas congeladas. Rev Argent Microbiol 38: 38-40.

21. Muñoz S, Vilca M, Ramos D, Lucas J. 2013. Frecuencia de enterobacterias en verduras frescas de consumo crudo expendidas en cuatro mercados de Lima, Perú. Rev Inv Vet Perú 24: 300-306.

22. Nilsson $O$. 2015. Hygiene quality and presence of ESBL-producing Escherichia coli in raw food diets for dogs. Infect Ecol Epidemiol 5: 28758. doi: 10.3402/ iee.v5.28758

23. Nuesch-Inderbinen M, Treier A, Zurfluh K, Stephan R. 2019. Raw meat-based diets for companion animals: a potential source of transmission of pathogenic and antimirobial-resistant Enterobacteriaceae. Roy Soc Open Sci 6: 191170. doi: 10.1098/rsos.191179

24. Ripodas A, Fernández D, Macho M. 2017. Investigación de Escherichia coli productor de toxinas Shiga (STEC) en carnes y derivados cárnicos. Rev Sanid Milit 73: 147-152. doi: 10.4321/S188785712017000300002

25. Rodríguez-Angeles G 2002. Principales características y diagnóstico de los grupos patógenos de Escherichia coli. Salud Pública Mex 44: 464-475.

26. Ruiz-Roldán L, Martínes-Puchol S, Gomes C, Palma $N$, Riveros $M$, Ocampo K, et al. 2018. Presencia de Enterobacteriaceae y Escherichia coli multirresistente a antimicrobianos en carne adquirida en mercados tradicionales en Lima. Rev Peru Med Exp Salud Pública 35: 425-432. doi: 10.17843/ rpmesp.2018.353.3737
27. Saeedi P, Yazdanparast M, Behzadi E, Hatef A, Latif S, Nazarian S. 2017. A review on strategies for decreasing $E$. coli $\mathrm{O} 157: \mathrm{H} 7$ risk in animals. Microb Pathogenesis 103: 186-195. doi: 10.1016/ j.micpath.2017.01.001

28. Sandri M, Dal Monego S, Conte G, Sgorlon S, Stefanon B. 2017. Raw meat-based diet influences faecal microbiome and end products of fermentation in healthy dogs. BMC Vet Res 13:65. doi: 10.1186/s12917-0170981-z

29. Schlesinger DP, Joffe D. 2011. Raw food diets in companion animals: a critical review. Can Vet J 52: 50-54.

30. Stockman J, Fascetti AJ, Kass PH, Larsen JA. 2013. Evaluation of recipes of home-prepared maintenance diets for dogs. J Am Vet Med Assoc 242: 15001505. doi: 10.2460/javma.242.11.1500

31. Strohmeyer RA, Morley PS, Hyatt DR, Dargatz DA, Scorza V, Lappin MR. 2006. Evaluation of bacterial and protozoal contamination of commercially available raw meat diets for dogs. $\mathrm{J}$ Am Vet Med Assoc 228: 537-542. doi: 10.2460/javma.228.4.537

32. Thrusfield M. 2005. Veterinary epidemiology. $2^{\text {nd }}$ ed. Oxford: Blackwell Science. 488 p.

33. Van Bree FP, Bokken GC, Mineur R, Franssen F, Opsteegh M, van der Giessen J, et al. 2018. Zoonotic bacteria and parasites found in raw meatbased diets for cats and dogs. Vet Rec182: 50. doi: 10.1136/vr.104535

34. Weese JS, Rousseau J, Arroyo L. 2005. Bacteriological evaluation of commercial canine and feline raw diets. Can Vet J 46: 513-516.

35. Zotta CM, Lavayén S, Hollman P, Lanfranconi $V .2015$. Animales domésticos como reservorio de Escherichia coli productor de toxina shiga en Mar del Plata. J Selva Andina Res Soc 6: 2-9. doi: 10.36610/j.jsars.2015.060100002 